\title{
Modeling transient magnetohydrodynamic peristaltic pumping of electroconductive viscoelastic fluids through a deformable curved channel
}

\author{
V K Narla · Dharmendra Tripathi . \\ O Anwar Bég · A Kadir
}

the date of receipt and acceptance should be inserted later

\begin{abstract}
A mathematical model is presented to analyze the unsteady peristaltic flow of magnetized viscoelastic fluids through a deformable curved channel. The study simulates the bio-inspired pumping of electro-conductive rheological polymers which possess both electro-conductive and viscoelastic properties. The Jeffrey viscoelastic model is utilized which features both relaxation and retardation terms of relevance to real polymers. A magnetic body force is incorporated for the influence of static radial magnetic field. The mass and momentum conservation equations are formulated in a intrinsic coordinate system and transformed with appropriate variables into a non-dimensional system between the wave and the laboratory frames, under lubrication (i.e. low Reynolds number and long wavelength) approximations. Kinematic and no-slip boundary conditions are imposed at the channel walls. A magnetic body force is incorporated for the influence of static radial magnetic field in the primary momentum equation. An analytical approach is employed to determine closed-form solutions for stream function, axial pressure gradient and volumetric flow rate. Spatio-temporal plots for pressure distribution along the channel (passage) length are presented to study the influence of curvature parameter, relaxation to retardation time ratio (Jeffrey first viscoelastic parameter) and Hartmann number (magnetic field parameter). The effects of these parameters on radial velocity distributions are also visualized. Cases of trapping and reflux in a curved channel are discussed. Streamline distributions are included to study trapping phenomena and to investigate more closely the
\end{abstract}

Department of Mathematics

GITAM University, Hyderabad-502329, India

E-mail: vknarla@gmail.com

Department of Mechanical Engineering

Manipal University Jaipur, Rajasthan-303007, India

E-mail: dharmendra.tripathi@jaipur.manipal.edu

Mechanical and Aeronautical Engineering

Salford University, Newton Building, The Crescent, Salford, M54WT, England, UK. 
impact of curvature, magnetic field and viscoelastic properties on bolus evolution. The reflux or retrograde motion of the particles is studied by particle advection based on Lagrangian viewpoint. The simulations provide new insight into the mechanisms of pumping of electro-conductive non-Newtonian liquids in realistic geometries.

Keywords Peristalsis · Electro-conductive fluids · Viscoelasticity · Trapping • Reflux · Pressure distribution · Curvature · Magnetohydrodynamics

PACS $47 \cdot 87 \cdot 75$

Mathematics Subject Classification (2000) 76Z05 - 92C05 - 76W05

\section{Introduction}

Magnetic fluids constitute an exciting new development in materials engineering. These materials which include magnetic polymer gels exhibit excellent electro-conductive properties and a variety of other characteristics which allow them to be implemented in intelligent devices in a variety of industries including medical [20], chemical [43], powder technology [21] and aerospace [44], [46]. In the safe, efficient and cost-effective transport of magnetic fluids and other magnetic fluids, a variety of sophisticated pumping mechanisms have been developed. These different types of magnetic fluid pumps aim to minimize working parts, reduce maintenance costs and mitigate clogging problems associated with corrosion and in the case of hazardous fluids, to avoid possible explosions [8]. Examples include annular-linear-induction pumps, helical-rotor electromagnetic pumps, direct-current conduction and pulsed magnetic field pumps [48], direct ferrohydrodynamic pumping using traveling magnetic fields [26], hybrid magnetic pumps [14] and ferro-magnetic micro-pumps. A strong trend in recent years has been to deploy biological mechanisms in hydromagnetic pump designs. Nature provides many efficient and robust mechanisms which significantly improve on existing magneto-pump propulsion designs. Inspired by the excellent hydrodynamic efficiency of swimming animals, Atencia and Beebe [5] developed a vortex-based biomimetic magnetic pump in which local vortices are utilized to propel magnetic fluid through shallow fluidic networks, via a rotating magnetic field. Hesketh et al. [17] considered an alternative biological propulsion mechanism, namely micro-cilia generated pumping of magnetohydrodynamic fluids using micro-machined high aspect ratio cilia on the pump channel walls actuated magnetically to maximize efficiency. Many other efficient propulsion methods are present in nature and are reviewed lucidly by Bach et al. [6]. One of the most versatile of these propulsion mechanisms for both internal fluids (and also external locomotion) is peristalsis. Physiological peristalsis entails the conveyance of biological fluids via progressive periodic waves of contraction or expansion moving axially along a tube or channel. It relies on the deformability of the vessel walls (distensibility). Peristaltic propulsion arises in an astonishing range of applications including 
swallowing food through the oesophagus, movement of chyme in the gastrointestinal tract, transport of blood in small blood vessels, embryo transport in the uterus, sperm dynamics in the human reproductive tract and passage of urine from kidney to the bladder. Wu et al. [45] and Bar-Cohen [9] have demonstrated the great adaptability of peristalsis-based pump designs which employ electro-conductive fluids. Many analytical and computational studies of peristaltic flows have been reported since the late 1960s. These studies have utilized both Newtonian and non-Newtonian fluid models and have addressed many diverse applications. A good summary of Newtonian viscous, inertiafree peristaltic flows has been documented in Jaffrin and Shapiro [19], who also highlighted applications in finger pumps which use positive displacement to convey materials via compression waves. They also popularized the transformation from the laboratory (moving) frame to the wave (stationary) frame in peristaltic modelling. Dodds et al. [12] conducted an experimental study of digestive peristalsis observing that both the longitudinal and circular esophageal musculature contributed to peristaltic transport of a bolus through the esophagus. They also found that respiratory movement was synchronous with thoracic and diaphragmatic movement and alterations in intraluminal esophageal pressure. Computational simulation of Newtonian peristaltic flow was reported by Takabatake et al. [37] who employed a successive over relaxation technique with a finite difference algorithm to study two-dimensional pumping at intermediate Reynolds numbers, noting that the reflux phenomenon is not modified throughout the entire regime with Reynolds number. Further studies include Tang and Shen [38] who also considered heat transfer and Li and Brasseur [25] who addressed finite conduit geometries. Kumar and Naidu [23] employed an up-winding finite element method for numerical simulation of peristaltic pumping, observing that progressive waves with high amplitude and low wave numbers manifest in high shear stress variations. Pal and Basseur [30] conducted a theoretical lubrication-based study of esophagul peristalsis noting that the coordination of a wave of local longitudinal contractions and a peristaltic wave of circular muscle contractions offers significant mechanical and physiological advantages in human swallowing.

The above studies were confined to Newtonian viscous flows. However many working fluids in biological systems and industry (including magnetic fluids) exhibit strongly non-Newtonian characteristics. These require material models which can simulate a wide spectrum of features including viscosity variation, thixotropy, pseudo-plasticity, viscoplasticity, relaxation, retardation, viscoelasticity, elongation, micro-structure, slip, spurt etc. In recent years many investigators have considered non-Newtonian peristaltic fluid dynamics. Hariharan et al. [13] used Fourier series expansions to derive solutions for peristaltic pumping of both power-law and Bingham fluids, in a diverging tube with various wall wave forms (sinusoidal, multi-sinusoidal, triangular, trapezoidal and square waves). They computed pathline trajectories and observed the great sensitivity of reflux to amplitude ratio, power-law rheological index and wave forms. Tripathi and Bég [41] used the He homotopy perturbation 
method (HPM) to compute solutions for peristaltic transport of generalized Maxwell viscoelastic fluids in a Darcy-Brinkman porous medium, identifying that volumetric flow rate is reduced with increasing relaxation time (viscoelastic parameter) in the complete pumping region and the free pumping region, whereas it is enhanced in the co-pumping region. Kiran et al. [22] used Eringen's micropolar model to investigate peristaltic motion and hydrodynamic dispersion with homogenous/heterogeneous chemical reactions as a simulation of digestive transport. They noted that dispersion is inhibited with cross viscosity coefficient and both homogeneous and heterogeneous chemical reaction rates, whereas it is encouraged with increasing micropolar (vortex viscosity) and wall deformation effects.

The electrically-conducting nature of many physiological fluids (e.g. blood) and magnetic fluids has also stimulated interest in magnetohydrodynamic peristaltic hydrodynamics. This involves the interaction between peristaltic wavy transport and externally applied magnetic fields (static or oscillating) usually via the Lorentzian hydromagnetic body force. Applications are of interest in surgical blood flow control and also industrial pumping systems where improved regulation of flow rates can be achieved non-intrusively. Rao and Mishra [33] studied nonlinear peristaltic pumping, deriving a cubic relationship for pressure gradient and time rate of flux and that curvature can lead to the absence of shear stress at different locations on the walls. Usha and Rao [42] studied both inertial and wall curvature effects on peristaltic pumping of a two-fluid system, noting with greater curvature trapping in the core is divided splits into three eddies, a larger bolus with two small boluses on either side and that there is a reduction in trapped bolus volume with higher curvature. Tripathi et al. [40] presented analytical solutions for magnetized peristaltic pumping of a Jefferys viscoelastic liquid in a finite tube, evaluating the influence of viscoelastic relaxation time, retardation time and Hartmann number on pressure, local wall shear stress and mechanical efficiency and also considering a non-integral number of waves. Latif et al. [24] presented perturbation solutions for peristaltic magnetohydrodynamic flow and heat transport in a dissipative third order Reiner-Rivlin viscoelastic fluid in a symmetric channel, also addressing the influence of temperature-dependent properties on thermofluid characteristics. Bhatti et al. [11] analyzed the electromagnetic peristaltic flow of a two-phase Jefferys viscoelastic fluid in a two-dimensional porous medium channel with thermal radiation and convection effects. They observed that temperatures and pumping rates in the retrograde pumping region are decreased with greater particle volume fraction. They also noted that temperatures are boosted with thermal radiative flux and that both larger volume fraction and greater magnetic field reduces the magnitudes of trapped bolus whereas electrical field accelerates the axial flow. The above investigations have generally been confined to straight conduits. Most physiological tubular organs are curved in nature. Curvature is also present in many technological pumping systems in the nuclear and chemical engineering industries. In curved conduit transport, the direction of flow is modified continuously and 
furthermore curved channels/tubes provide greater length for the same diameter relative to straight conduits. They therefore feature strongly in biological systems where space may be restricted. The simulation of curvature effects in peristaltic transport is therefore essential to accurately assess actual pumping characteristics. One of the earliest investigations of curvature effects in peristaltic flow was presented by Jaffrin [19] who considered an infinite train of sinusoidal waves propagating along the walls of a two-dimensional tube, observing that pumping performance is enhanced with increasing wall curvature and that the range of trapping of boluses is widened with greater curvature but narrowed with inertial effects. Sato et al. [36] computed a linear pressure-flow characteristic for peristaltic transport in a two-dimensional curved channel. They noted that the steepness of pressure-flow characteristic is enhanced with greater channel curvature and that a weaker reflux is present at the inner wall compared with the outer wall. They further observed the emergence of a dual structure to the trapped bolus comprising two asymmetrical parts with the inner bolus component decreasing in magnitude with larger curvature and the opposite effect computed for the outer bolus component. Heat transfer analysis of incompressible viscous fluid flow in a curved channel undergoing peristalsis presented in references $([4,16])$. Ramanamurthy et al. [32] also considered heat transfer in transient peristaltic flow of a viscous fluid in a two-dimensional curved channel, deriving expressions for stream function, pressure distribution, shear stress, temperature, and coefficient of heat transfer and noting the deviation from symmetrical flow at the inner and outer channel walls owing to curvature effects. Tripathi et al. [39] investigated both analytically and computationally the peristaltic transport of Nakamura-Sawada bi-viscosity nonNewtonian fluid in a curved tube. Further studies of non-Newtonian transport in curved channels and pipes have been communicated by Ali et al. [2] for Carreau fluids and Norouzi et al. [29] for Oldroyd viscoelastic fluids with heat transfer. Magnetohydrodynamic flow in curved channels have also been addressed in recent years. Bakalis and Hatzikonstantinou [7] studied hydromagnetic flow of a liquid metal in a curved annular conduit. Bég et al. [10] and Hoque et al. [18] investigated curvature effects on magnetohydrodynamic blood flows in curved conduits using a spectral numerical code. They observed that with greater magnetic field and lower curvature the axial flow is observed to be displaced toward the center of the vessel with corresponding low fluid particle vorticity strengths. Magnetohydrodynamic peristaltic flows in curved channels have been examined by Hayat et al. [15] for third grade rheological fluids, Noreen et al. [35] for power-law shear-thinning fluids, Noreen et al. [34] for Carreau fluids and Noreen et al. [28] for nanoflids. Ali et al. [1] presented peristaltic flow of a viscoelastic fluid with Williamson fluid model in the presence of a radially imposed magnetic field in curved channels. In another paper of Ali et al. [27], peristaltic flow of Carreau fluid model was studied in a curved channel. They solved the flow governing equations numerically by using a finite difference method. These studies all confirmed the significant impact of transverse magnetic field, wall curvature and non-Newtonian characteristics 
on pressure development and velocity fields in peristaltic inertia-free flows.

In the current study we consider the magnetohydrodynamic peristaltic pumping of electro-conductive viscoelastic fluids in a curved channel under the action of a radial magnetic field. The Jeffrey non-Newtonian model is employed which simulates accurately complex viscoelastic effects. Closed-form solutions are derived for stream function, axial pressure gradient and volumetric flow rate. The effects of curvature parameter, relaxation to retardation time ratio (Jeffrey first viscoelastic parameter) and Hartmann number (magnetic body force parameter) are visualized and elucidated in detail. Bolus dynamics is also examined via streamline plots. Another interesting phenomenon in peristaltic transport, particle reflux is computed in the Lagrangian view point. The present analysis may provide further understanding of the complex dynamics of electro-conductive (magnetic) fluids in industrial bio-inspired pumping systems [31] and micro-distributed energy supply systems [47].

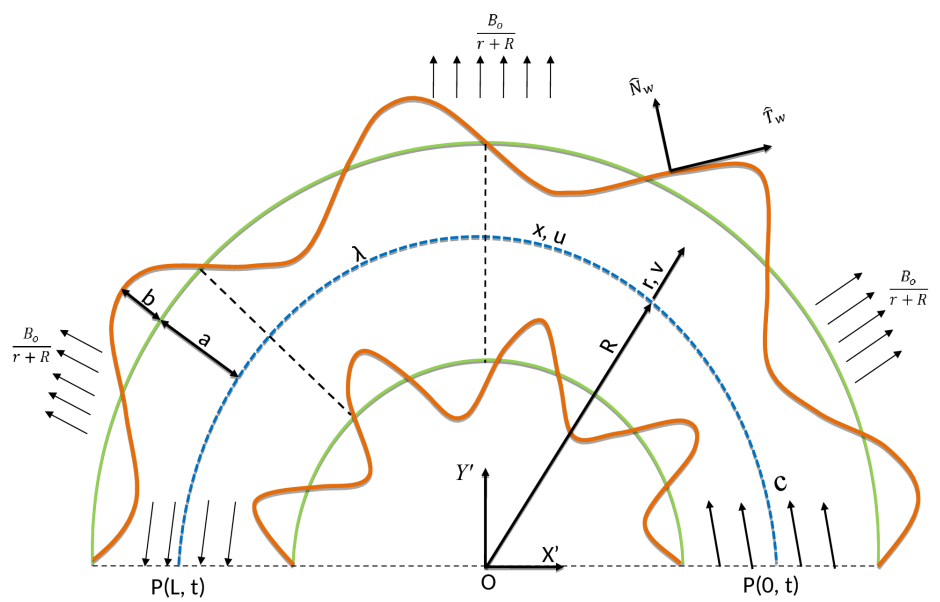

Fig. 1 Schematic representation of peristaltic flow of electro-conductive polymer under the influence of a radial magnetic field.

\section{Mathematical formulation and flow regime}

Consider the peristaltic pumping of an incompressible electro-conductive nonNewtonian fluid in a deformable curved channel with constant centerline radius $R$ and diameter $2 a$. A constant radial magnetic field is imposed. Magnetic induction and slip effects are neglected. It is convenient to select intrinsic coordinates $(x, r)$ defined with respect to rectangular cartesian coordinates 
$\left(X^{\prime}, Y^{\prime}\right)$. The geometry of the curved channel regime is depicted in Fig.1. The mathematical expressions for the coordinate transformations are given as:

$$
X^{\prime}=(R+r) \cos \left(\frac{x}{R}\right), \quad Y^{\prime}=(R+r) \sin \left(\frac{x}{R}\right) .
$$

The coordinate $\mathrm{x}$ lies along the center line of the curved channel, $\mathrm{r}$ is normal to it and is measured from the channel centre line. There is no component in the $z$ direction i.e. the flow is two-dimensional. The modified Navier-Stokes equations describing the flow in the laboratory frame of reference i.e. mass and momentum conservation equations in vectorial form with electromagnetic body force included, take the form :

$$
\begin{aligned}
\nabla \cdot \mathbf{V} & =0 \\
\rho\left(\frac{\partial}{\partial t}+\mathbf{V} \cdot \nabla\right) \mathbf{V} & =\nabla \cdot \mathbf{T}+\mathbf{J} \times \mathbf{B} .
\end{aligned}
$$

In the above equations $\mathbf{V}=u(x, r, t) \widehat{e}_{x}+v(x, r, t) \widehat{e}_{r}$ is the velocity vector, $\rho$ the density, $\mathbf{T}$ the stress tensor $(=-p \mathbf{I}+\mathbf{S}), p$ denote the pressure, $\mathbf{I}$ the identity tensor, $\mathbf{S}$ the extra stress tensor $\left(=\frac{\mu}{1+\lambda_{1}}\left(\dot{\gamma}+\lambda_{2} \ddot{\gamma}\right), \dot{\gamma}=\nabla \mathbf{V}+(\nabla \mathbf{V})^{T}, \ddot{\gamma}=\right.$ $\left.\frac{\mathrm{d}}{\mathrm{dt}} \dot{\gamma}\right), \mu$ the dynamic viscosity, $\lambda_{1}$ is the ratio of relaxation to retardation times, $\lambda_{2}$ is the retardation time and the dots over the quantities denote the material differentiation with respect to time. $\mathbf{J}$ the current density, $\mathbf{B}$ the magnetic field strength. The electromagnetic body force involved in eq. (3) under the assumption negligible magnetic Reynolds number and electrical field, can be obtained as:

$$
\mathbf{J} \times \mathbf{B}=-\sigma_{e}\left(\frac{B_{0}}{r+R}\right)^{2} u \widehat{e}_{x}
$$

in which $B_{0}$ is the constant magnitude of $\mathbf{B}$. The sinusoidal waves propagating along the channel walls are described as:

$$
\begin{gathered}
r=h(x, t)=a-b \cos ^{2}\left[\pi\left(\frac{x}{\lambda}-\frac{t}{T}\right)\right] \quad \text { (Upper wall) } \\
r=-h(x, t)=-a+b \cos ^{2}\left[\pi\left(\frac{x}{\lambda}-\frac{t}{T}\right)\right] \quad \text { (Lower wall). }
\end{gathered}
$$

Here, $b$ is the wave amplitude, $\lambda$ the wave length, $t$ the time, $T$ the wave period, and $h$ the radial displacement of the wave from the centerline. The wavelength is large compared with the channel's half width $(a<<\lambda)$. The governing equations with the associated boundary conditions for magnetohydrodynamic incompressible viscous Jeffrey fluid flow may be shown to reduce to:

$$
\begin{gathered}
\frac{R}{r+R} \frac{\partial u}{\partial x}+\frac{\partial v}{\partial r}+\frac{v}{r+R}=0 \\
\rho\left[\frac{\partial u}{\partial t}+(\mathbf{V} \cdot \nabla) u+\frac{u v}{r+R}\right]=-\frac{R}{r+R} \frac{\partial p}{\partial x}+\frac{R}{r+R} \frac{\partial \tau_{x x}}{\partial x} \\
+\frac{1}{(r+R)^{2}} \frac{\partial}{\partial r}\left\{(r+R)^{2} \tau_{r x}\right\}-\sigma_{e}\left(\frac{B_{0}}{r+R}\right)^{2} u
\end{gathered}
$$


$\rho\left[\frac{\partial v}{\partial t}+(\mathbf{V} \cdot \nabla) v-\frac{u^{2}}{r+R}\right]=-\frac{\partial p}{\partial r}-\frac{\tau_{x x}}{r+R}+\frac{R}{r+R} \frac{\partial \tau_{x r}}{\partial r}+\frac{1}{r+R} \frac{\partial}{\partial r}\left\{(r+R) \tau_{r r}\right\}$,

where

$$
\begin{gathered}
\mathbf{V} \cdot \nabla=\frac{R u}{(r+R)} \frac{\partial}{\partial x}+v \frac{\partial}{\partial r} \\
\tau_{x x}=\frac{2 \mu}{1+\lambda_{1}}\left[1+\lambda_{2}\left\{v \frac{\partial}{\partial r}+\frac{R u}{r+R} \frac{\partial}{\partial x}\right\}\right]\left(\frac{R}{r+R} \frac{\partial u}{\partial x}+\frac{v}{r+R}\right), \\
\tau_{x r}=\tau_{r x}=\frac{\mu}{1+\lambda_{1}}\left[1+\lambda_{2}\left\{v \frac{\partial}{\partial r}+\frac{R u}{r+R} \frac{\partial}{\partial x}\right\}\right]\left(\frac{\partial u}{\partial r}+\frac{R}{r+R} \frac{\partial v}{\partial x}-\frac{u}{r+R}\right), \\
\tau_{r r}=\frac{2 \mu}{1+\lambda_{1}}\left[1+\lambda_{2}\left\{v \frac{\partial}{\partial r}+\frac{R u}{r+R} \frac{\partial}{\partial x}\right\}\right] \frac{\partial v}{\partial x},
\end{gathered}
$$

The boundary conditions prescribed at the curved channel walls are:

(i). Kinematical boundary condition (the normal velocity of fluid at the wall is same as wall velocity),

$$
\bar{V} \cdot \widehat{N}_{W}=V_{W}
$$

(ii). No-slip boundary condition (the tangential velocity of the fluid and the wall agree),

$$
\bar{V} \cdot \widehat{T}_{W}=0
$$

where $\widehat{T}_{W}$ and $\widehat{N}_{W}$ are the unit tangent and normal vectors at the wall respectively. These vectors are defined as

$$
\widehat{T}_{W}=\frac{\widehat{e}_{x}+\frac{R}{R+r} \frac{\partial h}{\partial x} \widehat{e}_{r}}{\sqrt{1+\frac{R^{2}}{(R+r)^{2}}\left(\frac{\partial h}{\partial x}\right)^{2}}}, \widehat{N}_{W}=\frac{-\frac{R}{R+r} \frac{\partial h}{\partial x} \widehat{e}_{x}+\widehat{e}_{r}}{\sqrt{1+\frac{R^{2}}{(R+r)^{2}}\left(\frac{\partial h}{\partial x}\right)^{2}}} .
$$

The following dimensionless variables and parameter are introduced,

$$
\begin{gathered}
x^{\prime}=\frac{x}{\lambda}, r^{\prime}=\frac{r}{a}, u^{\prime}=\frac{u}{c}, v^{\prime}=\frac{v}{\delta c}, h^{\prime}=\frac{h}{a}, \phi=\frac{b}{a}, \kappa=\frac{R}{a}, t^{\prime}=\frac{t}{T}, L^{\prime}=\frac{L}{\lambda}, \\
p^{\prime}=\frac{a^{2} p}{\mu c \lambda}, R e=\frac{c a \delta}{\nu}, \psi^{\prime}=\frac{\psi}{a c}, F=\frac{Q}{a c}, H a^{2}=\frac{\sigma_{e} B_{0}^{2}}{\mu}, \tau_{i j}^{\prime}=\frac{a \tau_{i j}}{\mu c} .
\end{gathered}
$$

Here, $x^{\prime}$ is dimensionless axial coordinate, $r^{\prime}$ is dimensionless radial coordinate, $u^{\prime}$ is dimensionless axial velocity, $v^{\prime}$ is dimensionless radial velocity, $h^{\prime}$ is dimensionless radial displacement of the wave from the centerline, $\phi$ is the amplitude ratio, $\kappa$ is the curvature parameter, $t^{\prime}$ is non-dimensional time, $L^{\prime}$ is non-dimensional channel length, $p^{\prime}$ is non-dimensional pressure, $R e$ is Reynolds number, $\psi^{\prime}$ is non-dimensional stream function, $F$ is volume flow rate, $c=\frac{\lambda}{T}$ is the velocity of the wave, $\delta=\frac{a}{\lambda}$ defines wave number, $L$ is the length of the channel, $\mathrm{Ha}$ is Hartman number (ratio of magnetic body force and viscous hydrodynamic force), $\lambda_{1}$ is the ratio of relaxation to retardation times and $\tau_{i j}^{\prime}$ is dimensionless shear stress tensor. Eqs. (7)-(11) are therefore non-dimensionalized using eq. (12) and after dropping the primes, the reduced 
transport equations emerge as:

$$
\begin{gathered}
\frac{\kappa}{r+\kappa} \frac{\partial u}{\partial x}+\frac{\partial v}{\partial r}+\frac{v}{r+\kappa}=0 \\
\operatorname{Re} \delta\left[\frac{\partial u}{\partial t}+\frac{u \kappa}{r+\kappa} \frac{\partial u}{\partial x}+v \frac{\partial u}{\partial r}+\frac{u v}{r+\kappa}\right]=-\frac{\kappa}{r+\kappa} \frac{\partial p}{\partial x}+\delta \frac{\kappa}{r+\kappa} \frac{\partial \tau_{x x}}{\partial x} \\
+\frac{1}{(r+k)^{2}} \frac{\partial}{\partial r}\left\{(r+k)^{2} \tau_{r x}\right\}-H a^{2} \frac{u}{(r+\kappa)^{2}} \\
\operatorname{Re} \delta\left[\delta^{2}\left(\frac{\partial v}{\partial t}+\frac{u \kappa}{r+\kappa} \frac{\partial v}{\partial x}+v \frac{\partial v}{\partial r}\right)-\frac{u^{2}}{r+\kappa}\right]=-\frac{\partial p}{\partial r}-\delta \frac{\tau_{x x}}{r+R} \\
+\delta^{2} \frac{\kappa}{r+\kappa} \frac{\partial \tau_{x r}}{\partial r}+\frac{\delta}{r+\kappa} \frac{\partial}{\partial r}\left\{(r+\kappa) \tau_{r r}\right\}
\end{gathered}
$$

where

$$
\begin{gathered}
\tau_{x x}=\frac{2 \delta}{1+\lambda_{1}}\left[1+\frac{\lambda_{2} \delta c}{a}\left\{\frac{\partial}{\partial t}+\frac{\kappa u}{r+\kappa} \frac{\partial}{\partial x}+v \frac{\partial}{\partial r}\right\}\right]\left(\frac{\kappa}{r+\kappa} \frac{\partial u}{\partial x}+\frac{v}{r+\kappa}\right), \\
\tau_{x r}=\tau_{r x}=\frac{1}{1+\lambda_{1}}\left[1+\frac{\lambda_{2} \delta c}{a}\left\{\frac{\partial}{\partial t}+\frac{\kappa u}{r+\kappa} \frac{\partial}{\partial x}+v \frac{\partial}{\partial r}\right\}\right]\left(\frac{\partial u}{\partial r}+\delta^{2} \frac{\kappa}{r+\kappa} \frac{\partial v}{\partial x}-\frac{u}{r+\kappa}\right), \\
\tau_{r r}=\frac{2 \delta}{1+\lambda_{1}}\left[1++\frac{\lambda_{2} \delta c}{a}\left\{\frac{\partial}{\partial t}+\frac{\kappa u}{r+\kappa} \frac{\partial}{\partial x}+v \frac{\partial}{\partial r}\right\}\right] \frac{\partial v}{\partial x},
\end{gathered}
$$

The non-dimensionalized Kinematical and no-slip boundary conditions can be represented as

$$
\begin{array}{ccrl}
v-\frac{\partial h}{\partial x} u & =V_{W}\left[\left\{1+\delta^{2}\left(\frac{\kappa}{r+\kappa}\right)^{2}\left(\frac{\partial h}{\partial x}\right)^{2}\right\}^{\frac{1}{2}}\right], & & \text { at } r= \pm h \\
u+\delta^{2}\left(\frac{\kappa}{r+\kappa}\right) \frac{\partial h}{\partial x} v=0, & & \text { at } r= \pm h
\end{array}
$$

The stream function $\psi$ can be defined, which satisfies continuity eq. (7) is written as

$$
u=\frac{\partial \psi}{\partial r}, v=-\frac{\kappa}{r+\kappa} \frac{\partial \psi}{\partial x},
$$

Using eq. (18), the eqs. (13) to (15) can be written under the assumption of long wavelength and small Reynolds number approximation as

$$
\begin{gathered}
\frac{\kappa}{r+\kappa} \frac{\partial p}{\partial x}=\frac{1}{1+\lambda_{1}}\left\{\widetilde{\nabla}-\frac{1}{(r+\kappa)^{2}}\right\} \frac{\partial \psi}{\partial r}-\frac{H a^{2}}{(r+\kappa)^{2}} \frac{\partial \psi}{\partial r} \\
\frac{\partial p}{\partial r}=0
\end{gathered}
$$

where, $\widetilde{\nabla}=\frac{\partial^{2}}{\partial r^{2}}+\frac{1}{r+\kappa} \frac{\partial}{\partial r}$. The corresponding dimensionless boundary conditions are obtained by using the flux condition via continuity eq. (7)

$$
\frac{\partial \psi}{\partial r}=0, \text { at } r= \pm h(x, t)
$$




$$
\psi= \pm \frac{1}{2}\left[F(0, t)-2 \int_{0}^{x} \frac{\partial h}{\partial t}(s, t) d s\right], \text { at } r= \pm h(x, t)
$$

where $F(0, t)$ is the volume flow rate at channel inlet.

\section{Analytical solutions}

The stream function can be obtained by eliminating the pressure term from the equations (19) and (20). The obtained boundary value problem is solved with the boundary conditions consisting of the equations (21) to (22):

$$
\begin{aligned}
& \psi(x, r, t)=\frac{\partial p}{\partial x} \frac{\kappa\left(1+\lambda_{1}\right)^{-2}}{2 F_{2} H a^{4}}\left[4 h \kappa F_{1} N+F_{2}\left(2+H a^{2}\left(1+\lambda_{1}\right)\right)\left(h^{2}+\kappa^{2}\right)\right. \\
& \quad-F_{2}(r+\kappa)^{2}-2(1+N)\left(\kappa F_{3}+h F_{4}\right)\left(\kappa^{2}-h^{2}\right)^{N}(r+\kappa)^{1-N} \\
& \left.\quad-2(N-1)\left(-\kappa F_{3}+h F_{4}\right)(r+\kappa)^{1+N}\right]
\end{aligned}
$$

where the following definitions apply:

$$
\begin{gathered}
N=\sqrt{1+H a^{2}\left(1+\lambda_{1}\right)} \\
F_{1}=(-h+\kappa)^{2 N}+(h+\kappa)^{2 N}, F_{2}=(-h+\kappa)^{2 N}-(h+\kappa)^{2 N} \\
F_{3}=(-h+\kappa)^{N}-(h+\kappa)^{N}, F_{4}=(-h+\kappa)^{N}+(h+\kappa)^{N}
\end{gathered}
$$

Moreover, the axial pressure gradient can be obtained from equations (19) and (23) as:

$$
\frac{\partial p}{\partial x}(x, t)=\frac{H a^{4}\left(1+\lambda_{1}\right)^{2} F_{2}\left[H(t)+2 \int_{0}^{x} \frac{\partial h}{\partial t} d s\right]}{2 \kappa\left[\left(\kappa^{2} F_{3}^{2}+h^{2} F_{4}^{2}\right) N+h \kappa F_{2}\left(2+H a^{2}\left(1+\lambda_{1}\right)\right)\right]},
$$

where $H(t)=-F(0, t)$. Integrating equation (24) between 0 to $x$ yields the intraluminal pressure distribution function in the form:

$$
\Delta P(t, x)=\frac{H a^{4}\left(1+\lambda_{1}\right)^{2}}{2 \kappa} \int_{0}^{x} \frac{F_{2}\left[H(t)+2 \int_{0}^{s} \frac{\partial h}{\partial t} d u\right]}{\left[\left(\kappa^{2} F_{3}^{2}+h^{2} F_{4}^{2}\right) N+h \kappa F_{2}\left(2+H a^{2}\left(1+\lambda_{1}\right)\right)\right]} .
$$

The integrating constant function $H(t)$ is determined by evaluating (25) at $x=L$ and is given by

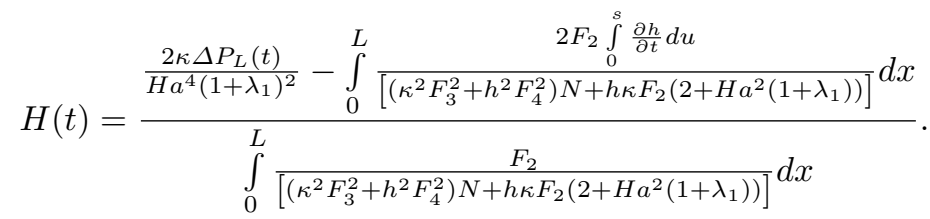


Here $\Delta P_{L}(t)=p(L, t)-p(0, t)$ is the pressure difference across the channel ends.

The transformations between the wave and the laboratory frames, in dimensionless form, are given by :

$$
X=x-t, Y=r, U=u-1, V=v, q=F-2 h, \Psi=\psi-r
$$

where the left hand side parameters are in the wave frame and the right hand side parameters are in the laboratory frame.

Averaging the volumetric flow rate along time period $T$ gives

$$
Q_{T}=\frac{1}{T} \int_{0}^{T} F(x, t) d t=\frac{1}{T} \int_{0}^{T}(q+2 h) d t=q+2-\phi
$$

From the equation (28), the axial pressure gradient in terms of time-averaged flow can be presented in the form:

$$
\frac{\partial p}{\partial x}(x, t)=-\frac{-H a^{4}\left(1+\lambda_{1}\right)^{2} F_{2}\left(Q_{T}+2 h-2+\phi\right)}{2 \kappa\left[\left(\kappa^{2} F_{3}^{2}+h^{2} F_{4}^{2}\right) N+h \kappa F_{2}\left(2+H a^{2}\left(1+\lambda_{1}\right)\right)\right]} .
$$

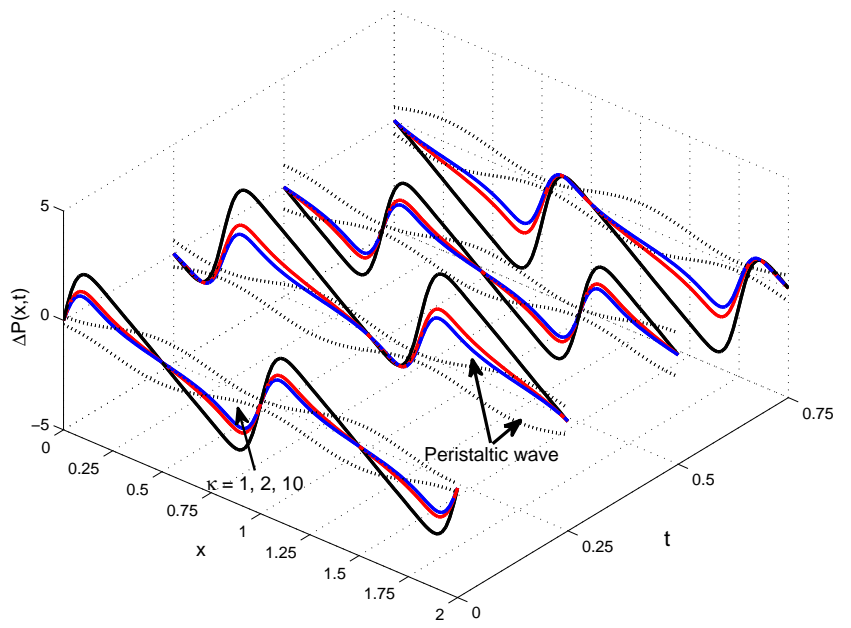

Fig. 2 Pressure distribution along the flow passage length for curved channel for $\phi=$ $0.8, L=2, H a=1, \lambda_{1}=1, \Delta P_{L}=0$. 


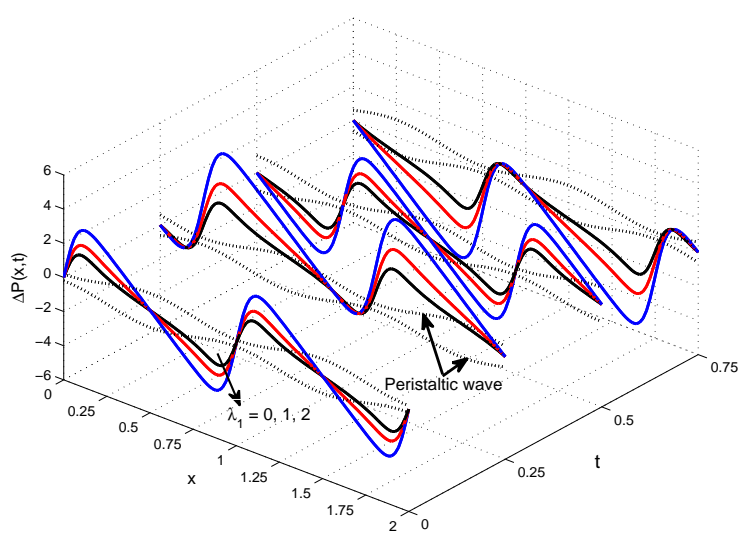

Fig. 3 Pressure distribution along the flow passage length of the curved channel for various viscoelastic parameters $\left(\lambda_{1}\right) \phi=0.8, \kappa=2, L=2, H a=1, \Delta P_{L}=0$.

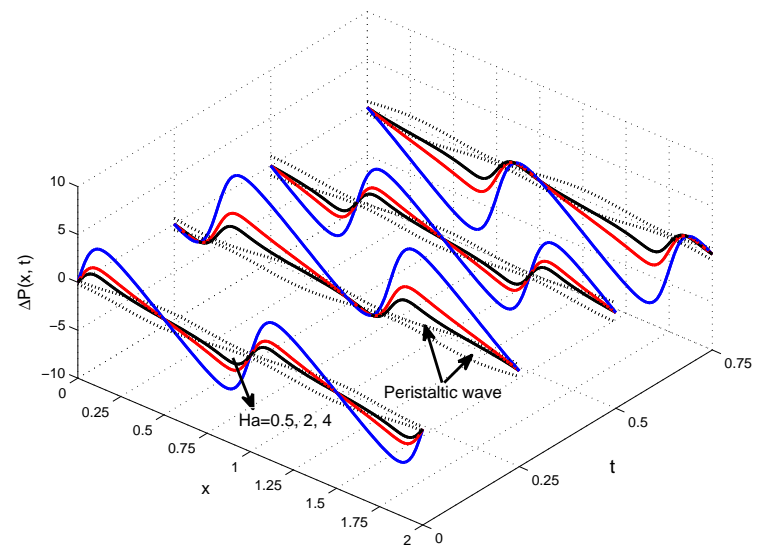

Fig. 4 Pressure distribution along flow passage length of the curved channel for various Hartmann numbers $(H a)$ with $\phi=0.8, \kappa=2, L=2, \lambda_{1}=1, \Delta P_{L}=0$.

\section{Computational results and discussion}

Numerical computations have been conducted in symbolic software based on the exact solutions derived in secn. 3. The influence of viscoelastic parameter $\left(\lambda_{1}\right)$, curvature parameter $(\kappa)$ and magnetic parameter i.e. Hartmann number $(H a)$ on pressure distributions (pressure difference across the channel length) in spatio-temporal $(x-t)$ plots, velocity variation with radial coordinate, stream lines in the wave frame (for bolus visualization) and particle advection (for reflux or retrograde motion of particles) are depicted in Figs. 2- 
13. Generally $\mathrm{Ha}$ is prescribed as unity implying an equivalence of Lorentzian magnetic drag force and viscous hydrodynamic force.

Fig. 2 illustrates the collective influence of curvature parameter $(\kappa)$, axial coordinate $(x)$ and time $(t)$ on local pressure difference across the channel passage length, $\Delta P(x, t)=p(x, t)-p(0, t)$. With greater curvature the peak pressure difference is significantly enhanced although it is reduced along the channel length from the entry to the termination. The undulating nature of the flow is clearly captured in the plot. Pressure difference magnitudes are generally enhanced with progression of time, when intermediate amplitude ratio is imposed $(\phi=0.8)$. The response in curvature variation is in agreement with other studies including Ali et al. [3] and Noreen et al. [35].The case of a straight channel is retrieved by setting $\kappa \rightarrow \infty$ i.e. infinite curvature. Pressure difference has also been shown to be enhanced with greater curvature parameter by Sato et al. [36] although only for the Newtonian case which they examined.

Fig. 3 demonstrates that with increasing viscoelastic parameter $\left(\lambda_{1}\right)$, there is generally an increase in the pressure difference computed. Again as in fig. 2 the peristaltic waves are mapped onto the pressure difference profiles and appear as dotted lines. The case of a Newtonian fluid is retrieved when relaxation and retardation times are neglected in the Jeffrey model $\left(\lambda_{1}=0\right)$ and this achieves lower pressure differences. The electro-conductive polymer is therefore assisted to pressure build up in peristaltic wave propagation. Relaxation time is an important parameter in polymer dynamics and relates to the time required for polymer chains to return to equilibrium after experiencing a disturbance e.g. pressure, shock etc. The retardation time is also incorporated in the 1 parameter (denominator) but relates more to the delay in elastic response to pressure in the viscoelastic fluid (this delay is absent in elastic materials which respond instantaneously to a deformation). When $\lambda_{1}=1$ both relaxation and retardation times are the same. For $\lambda_{1}>1$, the electro-conductive polymer takes much longer to return to the equilibrium state than it takes to respond elastically. Again with progression in time significant amplification in pressure difference is observed. Pressure peaks are also significantly displaced away from the channel inlet i.e. with increasing values of axial coordinate $(x)$.

Fig. 4 shows that the Hartmann number, which simulates the influence of radial magnetic field also modified the pressure difference plots markedly. Increasing Hartmann number evidently enhances pressure difference magnitudes throughout the length of the finite channel. The Lorentzian magnetohydrodynamic force associated with the radial magnetic field acts transverse to the radial direction and impedes flow in the axial direction. This manifests in higher pressure differences since the flow is inhibited significantly with radial magnetic field. The imposition of a magnetic field therefore exerts a tangible effect on the peristalsis process. Magnitudes of pressure difference are also substantially boosted initially with elapse in time but for higher times they are reduced. Furthermore peak pressure differences are larger for a short distance 


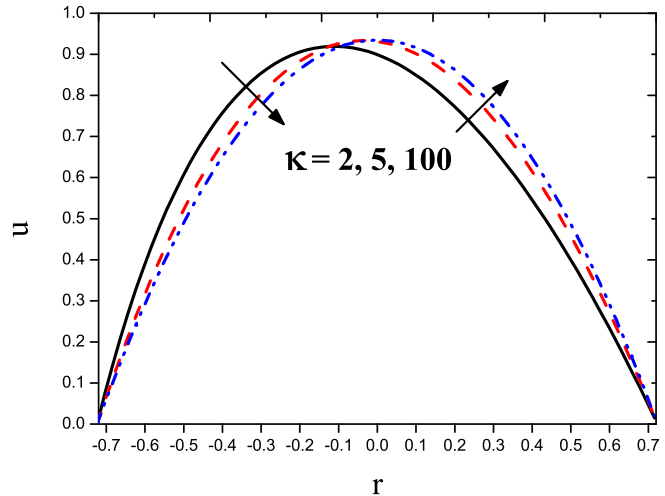

Fig. 5 Axial velocity profiles for different values of curvature parameter $(\kappa)$ with $\phi=$ $0.8, \lambda_{1}=0, H a=1, x=0.3, t=0, \Delta P_{1}=0$.

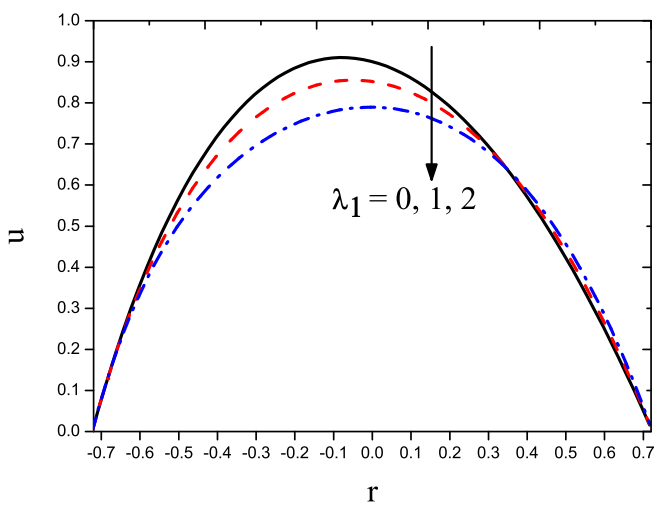

Fig. 6 Axial velocity profiles for different values of viscoelastic parameter $\left(\lambda_{1}\right)$ with $\phi=$ $0.8, \kappa=2, H a=2, x=0.3, t=0, \Delta P_{1}=0$.

into the channel and thereafter are in-fact slightly reduced. With intermediate times, the troughs are significantly suppressed whereas the peaks are amplified although as noted earlier, with further progression in time this response is eliminated. The pressure difference response to coordinate and time variation is therefore not consistent and is continuously altered.

Figs. 5-7 present the influence of curvature parameter $(\kappa)$, viscoelastic parameter $\left(\lambda_{1}\right)$ and Hartmann number $(\mathrm{Ha})$, respectively on axial velocity evolution across the channel radial span $(-0.72<r<0.72)$. Different responses are computed in the different channel half spans in all these plots. Fig. 5 shows that in the lower (inner) half-space increasing curvature is observed to de- 


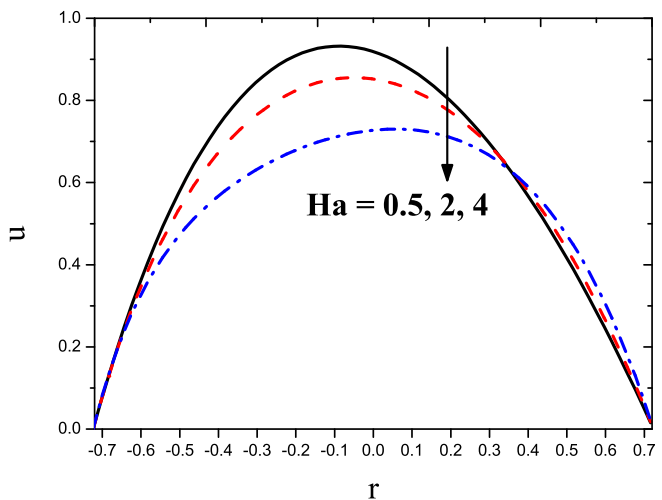

Fig. 7 Axial velocity profiles for different values of Hartmann number $(\mathrm{Ha})$ with $\phi=$ $0.8, \kappa=2, \lambda_{1}=1, x=0.3, t=0, \Delta P_{1}=0$.

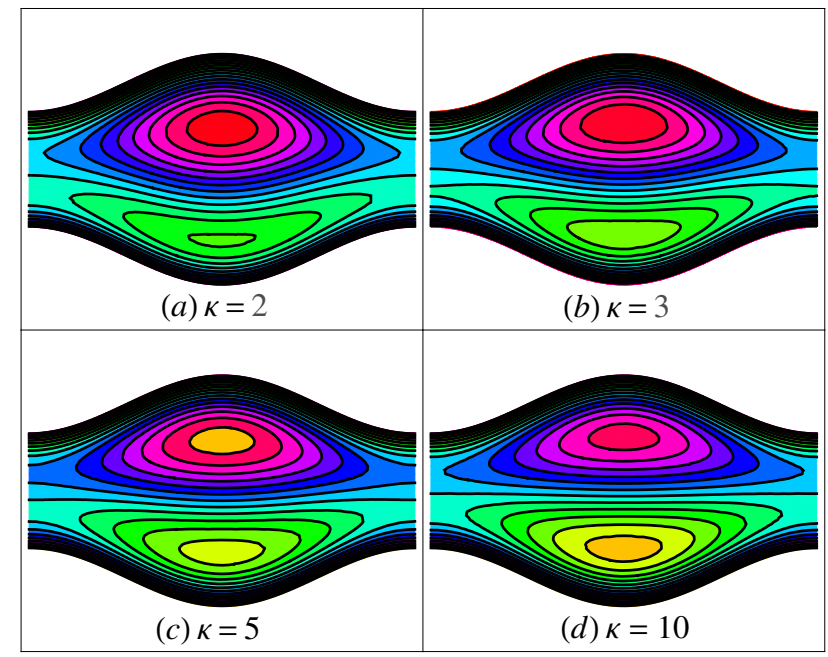

Fig. 8 Streamlines in the wave frame for different curvature parameters $\kappa$ with $\phi=$ $0.5, \lambda_{1}=1, H a=0.01, Q_{T}=1.75$.

celerate the flow whereas in the outer channel half space there is a significant acceleration. The velocity distribution is therefore asymmetric across the channel and this trend has been confirmed by many investigators including Jaffrin [19], Rao and Mishra [33] and more recently by Hayat et al. [15]. Symmetry can be achieved only for the case of infinite curvature i.e. a straight channel 


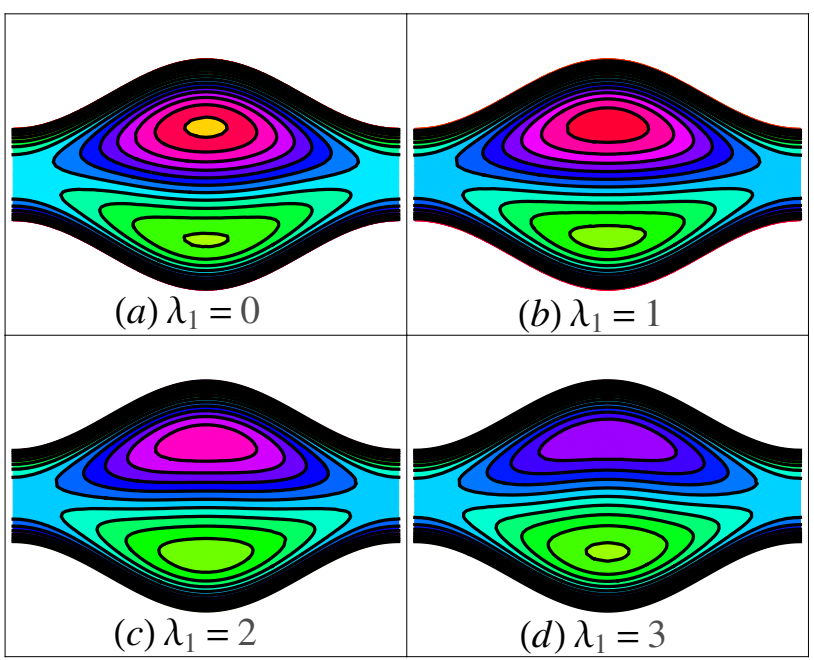

Fig. 9 Streamlines in the wave frame for Newtonian fluid $\left(\lambda_{1}=0\right)$ and Jeffrey's viscoelastic fluid $\left(\lambda_{1}>0\right)$ with $\phi=0.6, \kappa=3, H a=2, Q_{T}=1.75$.

setting $(\kappa \rightarrow \infty)$. Slightly higher magnitudes of velocity are observed in the outer channel half space than the inner channel half space, in the core zone of the channel. Fig. 6 indicates that with increasing viscoelastic parameter, there is a significant retardation in the channel flow throughout the inner channel half space and fore some distance into the outer channel half space region. In this zone Newtonian fluid achieves substantially greater velocity magnitudes. However as we approach the outer channel wall, a weak acceleration in the peristaltic axial flow is computed which thereafter effectively vanishes at the outer channel wall. The asymmetry in velocity distribution is again apparent and is a familiar characteristic of curved channel walls. For the majority of the channel regime, generally stronger viscoelasticity (higher relaxation time) which is usually associated with denser and more elastic effects in fluids generates flow deceleration. Non-Newtonian characteristics therefore impact considerably on the peristaltic propulsion characteristics, although even very strong viscoelastic fluids do not experience any flow reversal (velocity magnitudes are never negative). Fig. 7 exhibits the response in axial velocity to a change in Hartmann number. The profiles are similar to those computed for the viscoelastic effect. However a more dramatic reduction in velocity is caused by increasing radial magnetic field contribution compared with increasing relaxation time of the magnetic polymer. For $\mathrm{Ha}=0.5$ the viscous force in the regime is double that of the Lorentzian magnetic drag and for this scenario the axial velocity is maximized. For $\mathrm{Ha}=2$ and 4 , the Lorentzian force is respectively double 


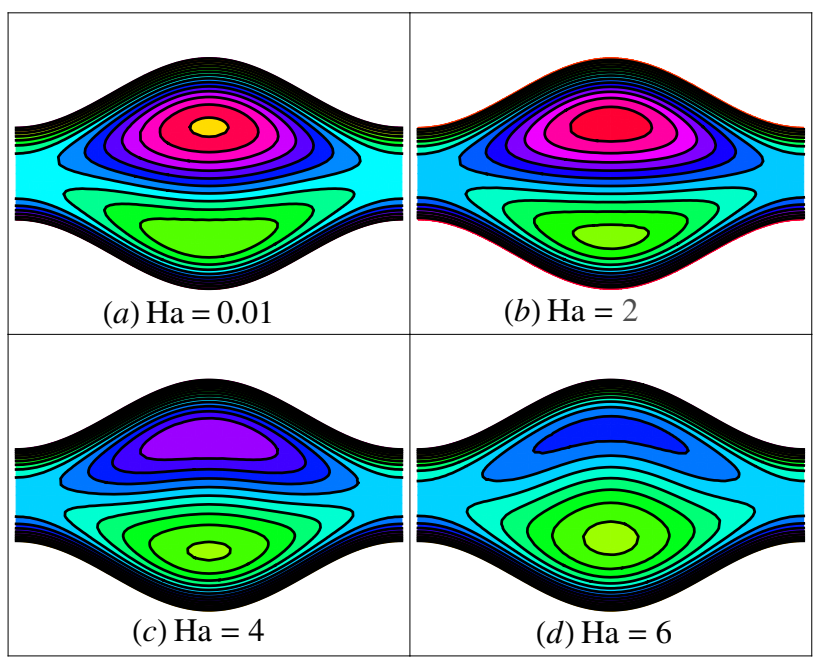

Fig. 10 Stream lines in the wave frame for various Hartmann numbers $(H a)$ with $\phi=$ $0.6, \kappa=3, \lambda_{1}=1, Q_{T}=1.75$.

and then quadruple that of the viscous force and noticeable deceleration is induced. However this is confined again to the inner channel half space and only for a short excursion into the outer channel half space. The reverse effect is manifested with further progression into the outer channel half space and clearly axial flow is found to be accelerated near the outer channel wall with higher Hartmann number. Asymmetric flow is therefore again apparent and the impact of radial magnetic field is found to be influenced by spatial location in the channel.

Figs. 8-10 depict the effects of curvature parameter $(\kappa)$, viscoelastic parameter $\left(\lambda_{1}\right)$ and Hartmann number $(\mathrm{Ha})$, on the trapping of boluses in the wave frame visualized via streamline distributions. With increasing curvature of the channel (figs. 5-7), the two asymmetric boluses in the inner and outer channel half spaces are modified substantially. The outer channel bolus is decreased in magnitude whereas the inner channel bolus is found to grow significantly in size with greater curvature. The larger the curvature the closer the channel approximates the straight channel case. The infinite curvature parameter case implies an infinite channel radius i.e. the straight channel case. Therefore small values of curvature parameter imply a more curved channel. A stronger bolus is therefore present in the outer channel half space with lower curvature parameter and this is depleted with higher curvature parameter. Fig. 9 shows that with the Newtonian case $\left(\lambda_{1}=0\right)$ a smaller bolus is present in both the 


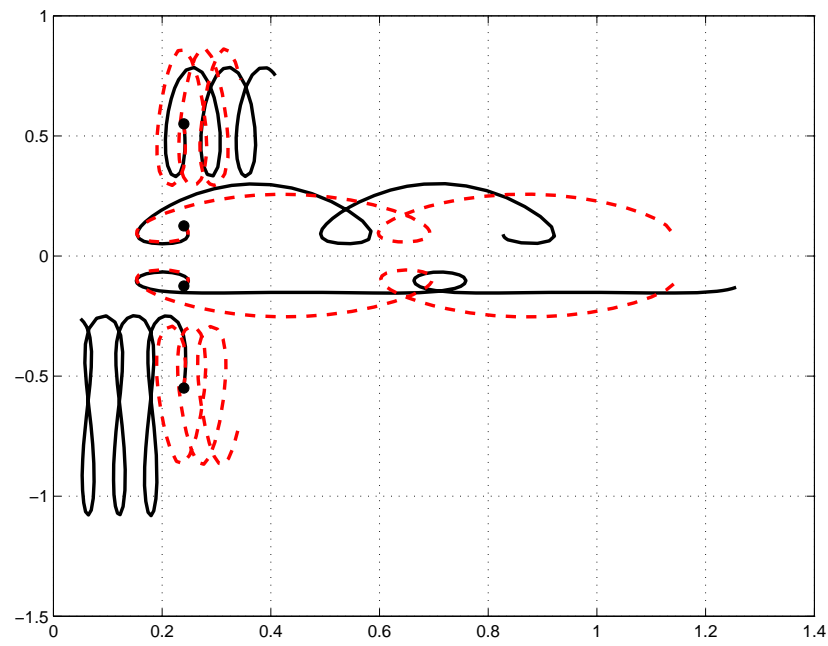

Fig. 11 Particle trajectories for $\kappa$ with $\phi=0.6, Q_{T} / Q_{T, M a x}=0.25, H a=0.5, \lambda_{1}=0$. Initially the fluid particles are located at $(0.24,0.125),(0.24,0.55)$. Solid lines for curved channel $\kappa=2$, dotted lines for straight channel $\kappa \rightarrow \infty$.

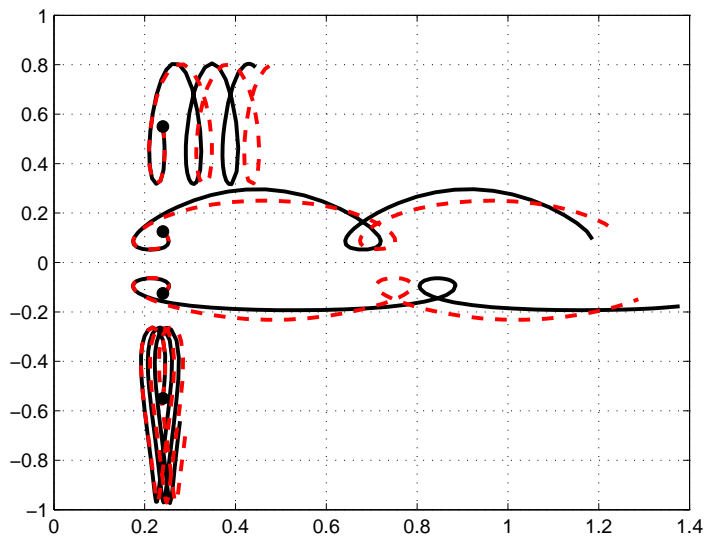

Fig. 12 Particle trajectories for $\lambda_{1}$ with $\phi=0.6, Q_{T} / Q_{T, \operatorname{Max}}=0.3, \kappa=3, \mathrm{Ha}=2$. Initially the fluid particles are located at $(0.24,0.125),(0.24,0.55)$. Solid lines for $\lambda_{1}=0$; Dotted lines for $\lambda_{1}=2$.

upper (outer) and lower (inner) channel half spaces. With increasing viscoelastic effect the upper bolus is observed to grow continuously whereas the lower bolus is initially enlarged and thereafter diminished again in magnitude. An increase in Hartmann number $(\mathrm{Ha})$, as observed in Fig. 10 is found to initially increase the upper bolus magnitude significantly and to initially decrease the size of the lower bolus. However for the highest Hartmann number $(\mathrm{Ha}=6)$ the effect is reversed and the upper (outer) bolus shrinks markedly whereas 


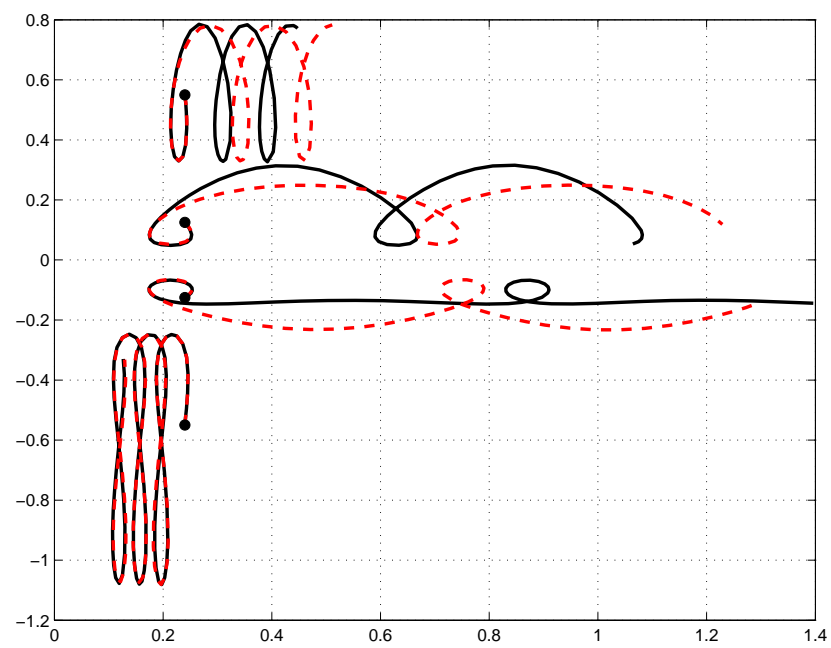

Fig. 13 Particle trajectories for $H a$ with $\phi=0.6, Q_{T} / Q_{T, M a x}=0.3, \kappa=2, \lambda_{1}=0$ Initially the fluid particles are located at $(0.24,0.125),(0.24,0.55)$. Solid lines for $H a=0.1$ and dotted lines for $H a=2$.

the lower bolus expands. A critical radial magnetic field therefore exists below and above which different responses are induced in the dual bolus structures in the inner and outer channel half space zones.

Particle retrograde motion or reflux can be computed by particle advection.

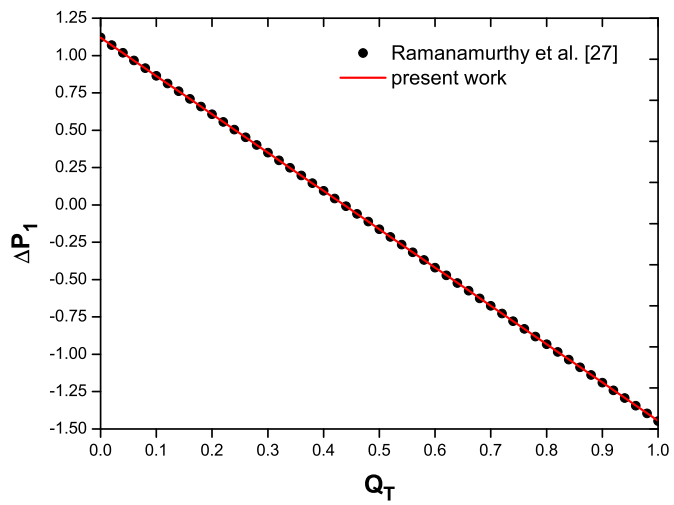

Fig. 14 Pressure rise per wavelength result validation with $\phi=0.4, \kappa=2$.

It is a process by which a massless particle is moving with the flow during the time the peristaltic wave completes one full cycle. Such Lagrangian trajectory of the massless fluid particle can be modeled by velocity field $\mathbf{V}(\mathbf{X}, \mathbf{t}) \in \mathbb{R}^{n}$. Consider the initial position of the massless particle is $\mathbf{X}_{\mathbf{0}}$ at time $t=t_{0}$, 


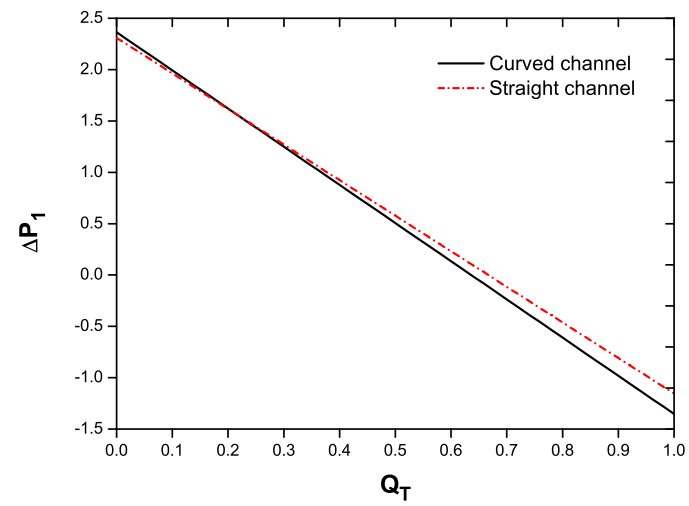

Fig. 15 Pressure rise per wavelength for variation in $\kappa$ with $\phi=0.4, \lambda=1, H a=1$.

then the resulting particle advection is specified by the initial value problem (IVP) $\dot{\mathbf{X}}=\mathbf{V}(\mathbf{X}, \mathbf{t}), \mathbf{X} \in \mathbb{R}^{n}, t \geq t_{0}$ subject to $\mathbf{X}=\mathbf{X}_{0}$ at $t=t_{0}$. The fluid particle trajectory may be computed by integrating these advection equations using a numerical ODE integrator, such as Runge-Kutta method. Figs. (11-13) demonstrate the particle trajectories for curvature parameter $(\kappa)$, viscoelastic parameter $\left(\lambda_{1}\right)$ and Hartmann number $(H a)$ respectively; particle initial locations are $x=0.24$ on $r=\{ \pm 0.125, \pm 0.55\}$, integration carried up to $T=3$. It has been observed that a massless particle positioned near the centre line of the channel undergoes net positive displacement. However, for the particles positioned away from the centre line travels lesser distance in longitudinal and greater distance in radial direction. The particle located near the lower wall have net negative displacement in curved channel $(\kappa=2)$. Whereas, in the case of straight channel $(\kappa \rightarrow \infty)$, almost symmetric motility of particle motion has been achieved as it shown in fig. 11. Particle motion for Newtonian fluid case $\left(\lambda_{1}=0\right)$ and for viscoelastic fluid case $\left(\lambda_{1}=2\right)$ in a curved channel $(\kappa=3)$ is shown in fig. 12. The particles located in the upper half space region, axial and radial motilities are constrained for Jeffrey viscoelastic fluid. Whereas, for Newtonian fluid case the particles in this region travels comparatively lesser distance before coming to rest. This happens because, the axial velocity component decreases radially and the particle trajectories become aligned towards the transverse axis direction for the case of Jeffrey viscoelastic fluid. There is no much influence on the particle reflex for both Newtonian and non-Newtonian cases. Fig. 13 shows the influence of radial magnetic field on particle motion in a curved peristaltic channel. The net axial positive mobility of the particle in the upper half space region and the positive mobility in axial direction of the particle in lower half space region decreases with an increase in $H a$. The particle trajectories for $H a$ are similar to those plotted for the viscoelastic effect. 
In order to compare the results, we have validated the pressure rise per one wave length $\left(\Delta P_{1}\right)$ against flow rate $\left(Q_{T}\right)$ for Newtonian fluid with almost negligible Hartmann number in a curved channel (i.e. $\lambda=0, H a=0.0001, \kappa=2$ ). We have observed that the results agree with the result obtained by Ramanamurthy et al. [32] (14). In the work of Ramanamurthy et al. [32], the hydrodynamics of peristaltic flow of Newtonian fluid without magnetic effect in a curved channel has been examined. In our current work, when the Jeffrey parameter $\left(\lambda_{1}=0\right)$ and Hartmann number $(\mathrm{Ha} \rightarrow 0)$ the scenario resembles the Newtonian fluid flow situation inside a peristaltic wavy channel. In fig. 14, we have compared these limiting cases. Fig. 15 displays the pressure rise per one wave length against flow rate for curved channel $(\kappa=2)$ and straight channel $(\kappa \rightarrow \infty)$ cases. The maximum pressure rise against which peristaltic movement generates fluid pumping increases with curvature of the channel. These findings agree with those of Ali et al. [4].

\section{Conclusions}

Motivated by simulating the peristaltic pumping of electro-conductive magnetic fluids in realistic geometries, a mathematical study has been presented for unsteady hydromagnetic peristaltic flow of viscoelastic fluids through a distensible curved channel under a radial magnetic field. Using lubrication approximations and transformations from the unsteady (laboratory) frame to the wave (steady) frame, the reduced Navier-Stokes equations have been solved under kinematic and no-slip boundary conditions at the channel walls. The computations have shown that:

1. With an increase in curvature parameter and time, peak pressure difference is increased whereas it is depressed with distance along the channel length.

2. With greater relaxation to retardation parameter and time, pressure difference is enhanced in the curved channel whereas it is reduced with distance from the channel inlet.

3. With increasing radial magnetic field i.e.Hartmann number, pressure difference is observed to consistently increase whereas with an increase in time there is an initial elevation in pressure difference and thereafter a reduction.

4. With increasing curvature parameter the peristaltic flow is decelerated in the lower (inner) half-space whereas it is accelerated in the outer channel half space and is therefore asymmetric across the channel span.

5. With increasing viscoelastic parameter, the flow is decelerated in inner channel half space and partially in the outer channel half space region, whereas it is accelerated near the upper (outer) channel wall.

6. With increasing Hartmann number there is a significant deceleration in the inner channel half space and again partially into the outer channel half space, whereas further towards the outer channel wall flow acceleration is induced. 
7. With increasing curvature parameter, the upper (outer) channel bolus is diminished with the opposite effect on the lower (inner) channel bolus which is amplified. Radial magnetic field therefore exerts a non-trivial influence on bolus formation.

8. With increasing relaxation to retardation ratio i.e. viscoelasticity, a strong increase in upper bolus size is induced whereas there is initially an expansion and thereafter a depletion in the lower bolus.

9. With increasing Hartmann number, the upper (outer) bolus grows in size whereas the lower bolus reduces in size. However with very high Hartmann number the opposite response is generated.

10. With increasing curvature of the channel, the particle reflex near the lower wall is enhanced. The influence of Jeffery parameter and Hartmann number on particle reflex is negligible at the prescribed initial location of particles.

The present study has identified some interesting magneto-fluid dynamic and rheological characteristics of magnetic polymer peristaltic dynamics in industriallyrelevant (curved) finite geometrical systems. Future investigations will consider more complex magnetic physics including electromagnetic induction, electrical fields and Maxwell displacement currents and will be communicated imminently.

\section{References}

1. Ali, N., Javid, K., Sajid, M.: Simulations of peristaltic slip-flow of hydromagnetic biofluid in a curved channel. AIP ADVANCES 6, 025,111(1-14) (2016)

2. Ali, N., Sajid, M., Abbas, Z., Javed, T.: Non-newtonian fluid flow induced by peristaltic waves in a curved chaneel. Eur. J. Mech-B/Fluids 5, 387-394 (2010)

3. Ali, N., Sajid, M., Hayat, T.: Long wave length flow analysis in a curved channel. Z. Naturforsch 65a, 191-196 (2010)

4. Ali, N., Sajid, M., Javed, T., Abbas, Z.: Heat transfer analysis of peristaltic flow in a curved channel. Int. J. Heat Mass Transfer 53, 3319-3325 (2010)

5. Atencia, J., Beebe, D.J.: Magnetically-driven biomimetic micro pumping using vortices. Lab Chip 4(6), 598-602 (2004)

6. Bach, D., Schmich, F., Masselter, T., Speck, T.: A review of selected pumping systems in nature and engineering-potential biomimetic concepts for improving displacement pumps and pulsation damping. Bioinspir. Biomim. 10, 051,001 (2015)

7. Bakalis, P.A., Hatzikonstantinou, P.M.: Effect of curvature and magnetic field on mhd flow of a liquid metal in a curved annular duct. Int. J. Numerical Methods for Heat and Fluid Flow 25(8), 1818-1833 (2015)

8. Baker, R.S., Tessier, M.J.: Handbook of Electromagnetic Pump Technology. Elsevier (1987)

9. Bar-Cohen, Y.: Artificial muscles using electroactive polymers (eap): capabilities, challenges and potential. Tech. rep., Biomimetics: Biologically Inspired Technologies (2005)

10. Bég, O.A., Hoque, M.M., Wahiuzzaman, M., Mahmud, M., Ferdows, M.: Spectral numerical simulation of laminar magneto-physiological dean flow. J. Mechanics in Medicine and Biology 14(3), (18 pages) (2014). DOI: 10.1142/ S021951941450047X

11. Bhatti, M.M., Zeeshan, A., Ijaz, N., Bég, O.A., Kadir, A.: Mathematical modelling of nonlinear thermal radiation effects on emhd peristaltic pumping of viscoelastic dusty fluid through a porous medium channel. Eng. Sci. Tech., Int. J. (2016). Doi.org/10.1016/j.jestch.2016.11.003

12. Dodds, W.J., Stewart, E.T., Hodges, D., Zhoralske, F.F.: Movement of the feline esophagus associated with respiration and peristalsis. Jounrnal of Clin. Invest. 52, 1-13 (1972) 
13. Hariharan, P., Seshadri, V., Banerjee, R.: Peristaltic transport of non-newtonian fluid in a diverging tube with different wave forms. Math Comput Model 48, 998-1017 (2008)

14. Hartshorne, H., Backhouse, C.J., Lee, W.E.: Ferrofluid-based microchip pump and valve. Sens. Actuators B 99, 592-600 (2004)

15. Hayat, T., Ali, N.: Peristaltic motion of a jeffrey fluid under the effect of a magnetic field in a tube. Nonlinear Sci. Numer. Simul. 13, 13431352 (2008)

16. Hayat, T., Javed, M., Hendi, A.A.: Peristaltic transport of viscous fluid in a curved channel with compliant walls. Int. J. Heat Mass Transfer 54, 1615-1621 (2011)

17. Hesketh, P., Hanasoge, S., Ballard, M., Erickson, M., Xu, J., Alexeev, A.: Pumping induced by bio-mimetic magnetic micro-cilia in creeping flows. PRiME 2016/230th Electrochemical Society (ECS) Meeting MA2016-02, 3822 (2016)

18. Hoque, M.M., Alam, M.M., Ferdows, M., Bég, O.A.: Numerical simulation of dean number and curvature effects on magneto-biofluid flow through a curved conduit. Proc. IMECHE- Part H; J. Engineering in Medicine 227(11), 1155-70 (2013)

19. Jaffrin, M.Y., Shapiro, A.H.: Peristaltic pumping. Ann. Rev. Fluid Mech. 3, 13-16 (1971)

20. Kalia, S., Kango, S., Kumar, A., Haldorai, Y., Kumari, B., Kumar, R.: Magnetic polymer nanocomposites for environmental and biomedical applications. Colloid. Polym. Sci. 292, 2025-2052 (2014)

21. Kim, S.H., Kim, S.J., Park, S.J., Mun, J.H., Kang, T.G., Park, J.M.: Rheological behavior of magnetic powder mixtures for magnetic pim. Korea Aust Rheol J 24, 121-127 (2012)

22. Kiran, G.R., Radhakrishnamacharya, G., , Bég, O.A.: Peristaltic flow and hydrodynamic dispersion of a reactive micropolar fluid: simulation of chemical effects in the digestive process. J. Mechanics in Medicine and Biology (2016)

23. Kumar, B.V.R., Naidu, K.B.: A numerical study of peristaltic flows. Comp. and Fluids 24, 161-176 (1995)

24. Latif, T., Alvi, N., Hussain, Q., Asghar, S.: Variable properties of mhd third order fluid with peristalsis. Results in Physics 6, 963-972 (2016)

25. Li, M., Brasseur, J.G.: Non steady peristaltic transport in finite length tubes. J. Fluid Mech. 248, 129-151 (1993)

26. Mao, L., Elborai, S., He, X., Zahn, M., Koser, H.: Direct observation of closed-loop ferrohydrodynamic pumping under traveling magnetic fields. Phys. Rev. B 84, 104,431 (2011)

27. N. Ali K. Javid, M.S., Bég, O.A.: Numerical simulation of peristaltic flow of a biorheological fluid with shear-dependent viscosity in a curved channel. Comput Methods Biomech Biomed Engin 19(6), 614-627 (2016)

28. Noreen, S., Qasim, M., Khan, Z.H.: Mhd pressure driven flow of nanofluid in curved channel. J. Magn. Magn. Mater. 393, 490-497 (2015)

29. Norouzi, M., Davoodi, M., Bég, O.A., Joneidi, A.: Analysis of the effect of normal stress differences on heat transfer in creeping viscoelastic dean flow. Int. J. Thermal Sciences 69, 61-69 (2013)

30. Pal, A., Brasseur, J.G.: The mechanical advantage of local longitudinal shortening on peristaltic transport. J. Biomech. Eng. 124, 94-100 (2002)

31. Philippova, O., Barabanova, A., Molchanov, V., Khokhlova, A.: Magnetic polymer beads: Recent trends and developments in synthetic design and applications. European Polymer Journal 47, 542-559 (2011)

32. Ramanamurthy, J.V., Prasad, K.M., Narla, V.K.: Unsteady peristaltic transport in curved channels. Phys. Fluids 25, 091,903(1-20) (2013)

33. Rao, A.R., Mishra, M.: Nonlinear and curvature effects on peristaltic flow of a viscous fluid in an asymmetric channel. ActaMechanica 168, 35-59 (2004)

34. S. Noreen, T.H., Alsaedi, A.: Flow of mhd carreau fluid in a curved channel. Applied Bionics and Biomechanics 10, 29-39 (2013)

35. S. Noreen, T.H., Alsaedi, A.: Magnetohydrodynamic peristaltic flow of a pseudoplastic fluid in a curved channel. Zeitschrift fur Naturforschung A 68a(5), 380-390 (2013)

36. Sato, H., Kawai, T., Fujita, T., Okabe, M.: Two dimensional peristaltic flow in curved channels. Trans. Jpn. Soc. Mech. Eng. Ser. B 66, 679-685 (2000)

37. Takabatake, S., Ayukawa, K.: Numerical study of two dimensional peristaltic flows. J. Fluid Mech. 122, 439-465 (1982) 
38. Tang, D., Shen, M.C.: Peristaltic transport of a heat conducting fluid subject to newton's cooling law at the boundary. Int. J. Engrg. Sci. 27, 809-825 (1989)

39. Tripathi, D., Akbar, N.S., Khan, Z.H., Bég, O.A.: Peristaltic transport of bi-viscosity fluids through a curved tube: a mathematical model for intestinal flow. Proc. IMechEPart H: J Engineering in Medicine (2016). DOI. 10.1177/095441 1916658318

40. Tripathi, D., Ali, N., dan M. K. Chaube, T.H., Hendi, A.A.: Peristaltic flow of mhd jeffrey fluid through finite length cylindrical tube. Appl. Math. Mech. -Engl. Ed. 32, 1231-1244 (2011)

41. Tripathi, D., Bég, O.A.: A numerical study of oscillating peristaltic flow of generalized maxwell viscoelastic fluids through a porous medium. Transport Porus Med 95, 337-348 (2012)

42. Usha, S., Rao, A.R.: Peristaltic transport of a biofluid in a pipr of elliptic cross section. J. Biomechanics 28, 45-52 (1995)

43. Watarai, H., Namba, M.: Magnetophoretic behavior of single polystyrene particles in aqueous manganese (ii) chloride. Anal Sci. 17(10), 1233-1236 (2001)

44. Wilson, M.J., Fuchs, M., Gordaninejad, F.: Characterization of magnetorheological polymer gels. J. Applied Polymer Science 84(14), 2733-2742 (2002)

45. Wu, Y., Zhou, D., Spinks, G.M., Innis, P.C., Megill, W.M., Wallace, G.G.: Titan: a conducting polymer based microfluidic pump. Smart Mater. Struct. 14, 1511-1516 (2005)

46. Xu, Y., Gong, X., Xuan, S.: Soft magnetorheological polymer gels with controllable rheological properties. Smart Mater. Struct. 22, 075,029 (2013)

47. Yamaguchi, H., Zhang, X.R., Higashi, S., Li, M.: Study on power generation using electro-conductive polymer and its mixture with magnetic fluid. J. Magn. Magn. Mater. 320, 1406-1411 (2008)

48. Youngquist, R., Lane, J., Immer, C., Simpson, J.: Pumping liquid oxygen by use of pulsed magnetic fields no moving parts are in contact with the oxygen. John F. Kennedy Space Center (2004) 